\title{
A New TCP for Persistent Packet Reordering
}

\author{
Stephan Bohacek, Member, IEEE, João P. Hespanha, Senior Member, IEEE, Junsoo Lee, \\ Chansook Lim, Student Member, IEEE, and Katia Obraczka, Member, IEEE
}

\begin{abstract}
Most standard implementations of TCP perform poorly when packets are reordered. In this paper, we propose a new version of TCP that maintains high throughput when reordering occurs and yet, when packet reordering does not occur, is friendly to other versions of TCP. The proposed TCP variant, or TCP-PR, does not rely on duplicate acknowledgments to detect a packet loss. Instead, timers are maintained to keep track of how long ago a packet was transmitted. In case the corresponding acknowledgment has not yet arrived and the elapsed time since the packet was sent is larger than a given threshold, the packet is assumed lost. Because TCP-PR does not rely on duplicate acknowledgments, packet reordering (including out-or-order acknowledgments) has no effect on TCP-PR's performance.

Through extensive simulations, we show that TCP-PR performs consistently better than existing mechanisms that try to make TCP more robust to packet reordering. In the case that packets are not reordered, we verify that TCP-PR maintains the same throughput as typical implementations of TCP (specifically, TCP-SACK) and shares network resources fairly. Furthermore, TCP-PR only requires changes to the TCP sender side making it easier to deploy.
\end{abstract}

Index Terms-Congestion control, packet reordering, transport protocols.

\section{INTRODUCTION}

$\mathbf{T}$ HE design of TCP's error and congestion control mechanisms was based on the premise that packet loss is an indication of network congestion. Therefore, upon detecting loss, the TCP sender backs off its transmission rate by decreasing its congestion window. TCP uses two strategies for detecting packet loss. The first one is based on the sender's retransmission timeout (RTO) expiring and is sometimes referred to as coarse timeout. When the sender times out, congestion control responds by causing the sender to enter slow-start, drastically decreasing its congestion window to one segment. The other loss detection mechanism originates at the receiver and uses TCP's sequence number. Essentially, the receiver observes the sequence numbers of packets it receives; a "hole" in the

Manuscript received October 12, 2003; revised May 3, 2004, and December 16, 2004; approved by IEEE/ACM TRANSACTIONS ON NETWORKING Editor N. Shroff. This work was supported in part by the National Science Foundation under Grants ANI-0322476 and CCR-0311084.

S. Bohacek is with the Department of Electrical and Computer Engineering, University of Delaware, Newark, DE 19716 USA (e-mail: bohacek@udel.edu).

J. P. Hespanha is with Department of Electrical and Computer Engineering, University of California, Santa Barbara, CA 93106 USA (e-mail: hespanha@ece.ucsb.edu)

J. Lee is with the Department of Computer Science, Sookmyung Women's University, Seoul 140-742, Korea (e-mail: jslee@ sookmyung.ac.kr).

C. Lim is with the Department of Computer Science, University of Southern California, Los Angeles, CA 90089 USA (e-mail: chansool@usc.edu).

K. Obraczka is with the Department of Computer Engineering, University of California, Santa Cruz, CA 95064 USA (e-mail: katia@cse.ucsc.edu).

Digital Object Identifier 10.1109/TNET.2006.873366 sequence is considered indicative of a packet loss. Specifically, the receiver generates a "duplicate acknowledgment" (or DUPACK) for every "out-of-order" segment it receives. Note that until the lost packet is received, all other packets with higher sequence number are considered "out-of-order" and will cause DUPACKs to be generated. Modern TCP implementations adopt the fast retransmit algorithm which infers that a packet has been lost after the sender receives a few DUPACKs. The sender then retransmits the lost packet without waiting for a timeout and reduces its congestion window in half. The basic idea behind fast retransmit is to improve TCP's throughput by avoiding the sender to timeout (which results in slow-start and consequently the shutting down of the congestion window to one).

Fast retransmit can substantially improve TCP's performance in the presence of sporadic reordering but it still operates under the assumption that out-of-order packets indicate packet loss and therefore congestion. Consequently, its performance degrades considerably in the presence of "persistent reordering." This is the case for reordering of both data and acknowledgment packets. Indeed, it is well known that TCP performs poorly under significant packet reordering (which may not be necessarily caused by packet losses) [1].

Packet reordering is generally attributed to transient conditions, pathological behavior, and erroneous implementations. For example, oscillations or "route flaps" among routes with different round-trip times (RTTs) are a common cause for out-oforder packets observed in the Internet today [2]. Internet experiments performed through MAE-East and reported in [3] show that $90 \%$ of all connections tested experience packet reordering. Researchers at SLAC performed similar experiments and found that $25 \%$ of the connections monitored reordered packets [4]. However, networks with radically different characteristics (when compared to the Internet, for example) can exhibit packet reordering as a result of their normal operation. This is the case of wireless networks, in particular multi-hop mobile ad-hoc networks (MANETs). In MANETs, which are also known as "networks without a network," there is no fixed infrastructure and every node can be source, sink, as well as forwarder of traffic. The potential for unconstrained mobility poses many challenges to routing protocols including frequent topology changes. Thus MANET routing protocols need to recompute routes often, which may lead to (persistent) packet reordering. In fact, improving the performance of TCP in such environments (by trying to differentiate out-of-order packets from congestion losses) has been the subject of several recent research efforts [5]-[7].

Mechanisms that provide different quality of service (QoS) by differentiating traffic may introduce packet reordering. An example of such mechanisms is DiffServ [8], which has been 
proposed to provide different QoS on the Internet. In the case of Expedited Forwarding, packets receive preferential treatment as long as the flow obeys negotiated bandwidth constraints. If the flow exceeds these constraints, the nonconformant packets are typically dropped. However, an alternative to dropping these packets is to lower their priority. In this case, the packets will be placed in different queues and will likely experience different latency, resulting in out-of-order delivery to the final destination. While this alternative is atypical, RFC 3246 simply specifies that packets should not be reordered, a weaker requirement than must not be reordered [38].

While packet reordering is often considered to be pathological in today's Internet, as shown in [3], it is actually part of normal operation for a number of routers containing parallel paths through the switch. Due to the scheduling algorithms used, different packet sizes and arrivals times may result in the reordering of packets entering on a single interface. While the exact cause of packet reordering lies in the details of the scheduling algorithm, a more general reason is that parallel paths are employed for economic reasons; it is cheaper to build multiple moderate speed paths than a single very high-speed path. The result of seeking this increase in cost efficiency is that packets may sometimes be reordered. TCP-PR is a transport protocol compatible with multipath routing, hence it will not limit the drive for efficiency at the lower layers.

Beyond router design, there are other areas that stand to gain efficiency if multiple paths are permitted. For example, load balancing is greatly simplified if single flows are permitted to use different paths. When a flow is restricted to use a single path, then optimal load balancing reduces to an NP-hard integer programming problem (cf. [9, p. 359]) but if the single path restriction is lifted, then optimal load balancing is a simpler linear programming problem. In [10], different flows may be split along multiple paths in order to meet QoS requirements. Permitting even single flows to be split results in a more efficient use of network resources. In the case of MANETs, spreading packets across different links also decreases the battery drain on any particular mobile node and may increase the lifetime of the network.

While efficiency is one area that may benefit from multipath routing, fault tolerance and security can also be improved by utilizing multiple paths. For example, in wired networks, multipath routing has been shown to reduce the impact of link failures [11]. Similarly, multipath routing can increase robustness to eavesdropping by spreading packets across different paths, thus forcing the attacker to sniff multiple links [11]. Multipath routing can take advantage of the considerable path redundancy that already exists in today's Internet. For example, in [12], it was shown that in the U.S. Sprintlink topology, 90\% of PoP pairs are connected through at least four distinct paths.

In MANETs, alternate path routing has been an active area of research. In [13], it is suggested that alternate paths be found and stored in an attempt to anticipate failures in the primary path. However, it was shown in [14] that alternate paths may grow stale and no longer exist when the primary path fails. One way to learn that alternate paths have failed is to send part of the data stream along them, as in multipath routing.

While multipath routing has many advantages, it leads to persistent packet reordering. Today's implementations of TCP are not compatible with networks that reorder packets and suffer great reductions in throughput when faced with persistent packet reordering. TCP's incompatibility with persistent packet reordering has been a major deterrent to the deployment of the mechanisms mentioned above on the Internet or on other networks in which TCP is prevalent. There are a number of methods for improving TCP's performance in packet-reordering prone environments, but most of them try to recover from occasional reordering and rely on the packet ordering itself to detect drops. However, under persistent reordering conditions, packet ordering conveys very little information on what is actually happening inside the network.

In this paper, we describe TCP-PR, a transport protocol that performs well under persistent packet reordering (Section III). The key feature of TCP-PR is that duplicate ACKs are not used as an indication of packet loss. Rather, TCP-PR relies exclusively on timeout. Both worst-case analysis and Internet traces are used to ensure that the timeout threshold is not too small and only actual packet losses cause retransmissions (Section IV). Through extensive ns-2 simulations, we evaluate the performance of TCP-PR, comparing it to a number of existing schemes that address TCP's poor performance under packet reordering (Section VI). We find that under persistent packet reordering, TCP-PR achieves significantly higher throughput. We also test TCP-PR's compatibility and fairness to standard TCP variants, specifically TCP-SACK (Section V). In the absence of packet reordering, TCP-PR is shown to have similar performance and competes fairly with TCP-SACK. TCP-PR neither requires changes to the TCP receiver nor uses any special TCP header option. Hence, TCP-PR is suitable for incremental deployment.

\section{RELATED WORK}

As previously mentioned, several mechanisms that address TCP's lack of robustness to packet reordering have been recently proposed. This section summarizes them and puts TCP-PR in perspective.

Upon detecting spurious retransmissions, the Eifel algorithm [15] restores TCP's congestion control state to its value prior to when the retransmission happened. The more spurious retransmissions of the same packet are detected, the more conservative the sender gets. For spurious retransmission detection, Eifel uses TCP's timestamp option and has the sender timestamp every packet sent. The receiver echoes back the timestamp in the corresponding acknowledgment (ACK) packets so that the sender can differentiate among ACKs generated in response to the original transmission as well as retransmissions of the same packet. ${ }^{1}$

DSACK [16] proposes another receiver-based mechanism for detecting spurious retransmission. Information from the receiver to the sender is carried as an option (the DSACK option) in the TCP header. The original DSACK proposal does not specify how the TCP sender should respond to DSACK notifications. In [1], a number of responses to DSACK notifications were proposed. The simplest one relies on restoring the

\footnotetext{
${ }^{1}$ As an alternative to timestamping every packet, Eifel can also use a single bit to mark the segment generated by the original transmission.
} 
sender's congestion window to its value prior to the spurious retransmission detected through DSACK. ${ }^{2}$ Besides recovering the congestion state prior to the spurious retransmission, other proposed strategies also adjust the DUPACK threshold (dupthresh). The different dupthresh adjustment mechanisms proposed include: 1 ) increment dupthresh by a constant; 2 ) set the new value of dupthresh to the average of the current dupthresh and the number of DUPACKs that caused the spurious retransmission; and 3) set dupthresh to an exponentially weighted moving average of the number of DUPACKs received at the sender. Recently, another scheme that relies on adjusting the dupthresh has been proposed [17].

Time-delayed fast-recovery (TD-FR), which was first proposed in [18] and analyzed in [1], addresses packet reordering. This method stands out from the others in that it utilizes timers as well as DUPACKs. It sets a timer when the first DUPACK is observed. If DUPACKs persists longer than a threshold, then fast retransmit is entered and the congestion window is reduced. The timer threshold is set to $\max (R T T / 2, D T)$, where $D T$ is the difference between the arrival of the first and third DUPACK.

A number of mechanisms to improve TCP's performance in MANET environments have been proposed. For example, TCP-DOOR [5] detects out-of-order packets by using additional sequence numbers (carried as TCP header options). To detect out-of-order data packets, the TCP sender uses a 2-byte TCP header option called TCP packet sequence number to count every data packet including retransmissions. For out-of-order DUPACK detection, the TCP receiver uses a 1-byte header option to record the sequence in which DUPACKs are generated. Upon detecting out-of-order packets (internally or informed by the receiver ${ }^{3}$ ), the TCP sender responds by either: (1) temporarily disabling congestion control for a given time interval (i.e., freezing the congestion control state, which includes the congestion window cwnd and the retransmission timer RTO), or (2) resetting the state to its value prior entering congestion avoidance. More recently, TCP-DCR [19], another variant of TCP for wireless networks, has been developed. Similarly to TD-FR, TCP-DCR delays response to DUPACKs. However, the delay of one RTT imposed by TCP-DCR is longer than that of TD-FR.

To some extent, the approaches described above still utilize packet ordering to detect drops. Indeed, when reordering is not persistent, packet ordering is still somewhat indicative of drops and therefore of congestion. However, if packets are persistently reordered, packet ordering conveys little information regarding congestion and thus should not be used to trigger congestion control. Consequently, as shown in Section VI, TD-FR and methods that use DSACK along with adjusting dupthresh perform poorly when faced with persistent packet reordering.

We propose to neglect DUPACKs altogether and rely solely on timers to detect drops: if the ACK for a packet has not arrived and the elapsed time since the packet is sent exceeds a threshold,

\footnotetext{
${ }^{2}$ Instead of instantaneously increasing the congestion window to the value prior to the retransmission event, the sender slow-starts up to that value in order to avoid injection of sudden bursts into the network.

${ }^{3} \mathrm{As}$ suggested in [5], the TCP receiver can notify the sender by setting a $O O O$ bit in the TCP ACK packet.
}

then the packet is assumed to be lost. In the next section we describe the TCP-PR algorithm in detail. There are two main design challenges in developing an adaptive timer threshold. First, the threshold must be chosen such that it is only surpassed when a packet has actually been lost. This is discussed in Section IV. The second challenge, covered in Section V, is to maintain fairness with current implementations of TCP. Section VI presents extensive simulation results that show that under persistent packet reordering, TCP-PR performs significantly better than existing packet reordering recovery methods.

\section{TCP-PR}

As mentioned above, the basic idea behind TCP-PR is to detect packet losses through the use of timers instead of duplicate acknowledgments. This is prompted by the observation that, under persistent packet reordering, duplicate acknowledgments are a poor indication of packet losses. Because TCP-PR relies solely on timers to detect packet loss, it is also robust to acknowledgment losses as the algorithm does not distinguish between data (on the forward path) or acknowledgment (on the reverse path) losses.

The proposed algorithms only require changes in the TCP sender and are therefore backward-compatible with any TCP receiver. TCP-PR's sender algorithm is still based on the concept of a congestion window, but the update of the congestion window follows slightly different rules than standard TCP. However, significant care was placed in making the algorithm fair with respect to other versions of TCP to ensure they can coexist.

\section{A. The Basic Algorithm}

Packets being processed by the sender are kept in one of two lists: the to-be-sent list contains all packets whose transmission is pending, waiting for an "opening" in the congestion window. The to-be-ack list contains those packets that were already sent but have not yet been acknowledged. Typically, when an application produces a packet it is first placed in the to-be-sent list; when the congestion window allows it, the packet is sent to the receiver and moved to the to-be-ack list; finally when an ACK for that packet arrives from the receiver, it is removed from the to-be-ack list (under cumulative ACKs, many packets will be simultaneously removed from to-be-ack). Alternatively, when it is detected that a packet was dropped, it is moved from the to-be-ack list back into the to-be-sent list.

As mentioned above, drops are always detected through timers. To this effect, whenever a packet is sent to the receiver and placed in the to-be-ack list, a timestamp is saved. When a packet remains in the to-be-ack list more than a certain amount of time it is assumed dropped. In particular, we assume that a packet was dropped at time $t$ when $t$ exceeds the packet's timestamp in the to-be-ack list plus an estimated maximum possible round-trip time mxrtt.

As data packets are sent and ACKs received, the estimate mxrtt of the maximum possible round-trip time is continuously updated. The estimate used is given by

$$
\operatorname{mxrtt}:=\beta \times \operatorname{srtt}
$$


where $\beta$ is a constant larger than 1 and srtt an exponentially weighted average of past RTTs. Whenever a new ACK arrives, we update srtt as follows:

$$
\operatorname{srtt}=\max \left\{\alpha^{\frac{1}{[\text { crnd }]}} \times \operatorname{srtt}, \text { sample }- \text { rtt }\right\}
$$

where $\alpha$ denotes a positive constant smaller than $1,\lfloor$ cwnd $\rfloor$ the floor of the current congestion window size, and sample - rtt the $R T T$ for the packet whose acknowledgment just arrived. ${ }^{4}$ The reason to raise $\alpha$ to the power $1 /\lfloor$ cwnd $\rfloor$ is that in one $R T T$ the formula in (2) is iterated $\lfloor$ cwnd $\rfloor$ times. This means that, e.g., if there were a sudden decrease in the $R T T$ then srtt would decrease by a rate of $\left(\alpha^{1 /\lfloor\text { cwnd }\rfloor}\right)^{\lfloor\text {cwnd }\rfloor}=\alpha$ per $R T T$, independently of the current value of the congestion window. The parameter $\alpha$ can therefore be interpreted as a smoothing factor in units of RTTs. As discussed in Section IV, the performance of the algorithm is actually not very sensitive to changes in the parameters $\beta$ and $\alpha$, provided they are chosen in appropriate ranges.

Note that srtt tracks the peaks of RTT. The rate that srtt decays after a peak is controlled by $\alpha$. The right-hand plot shows how large jumps can cause $R T T>\operatorname{mxrtt}$ (for this data set, occurrences at $15 \mathrm{~s}, 45 \mathrm{~s}, 75 \mathrm{~s}$, etc.) resulting in spurious timeouts (note that the jumps in RTT in the right-hand plot were artificially generated). In order for these jumps to cause a spurious timeouts, the jumps in RTT could occur no sooner than every 15 seconds. In this case, 1500 packets were delivered between these jumps. If the jumps occurred more frequently, then, as can be seen from the figure, mxrtt would not have decayed to a small enough value and spurious timeouts would not occur. Furthermore, if the jumps were larger, then the time between jumps to cause a timeout would be no smaller. The issue of spurious timeouts is closely examined in Section IV.

Two modes exist for the update of the congestion window: slow-start and congestion-avoidance. The sender always starts in slow-start and will only go back to slow-start after periods of extreme losses (cf. Section III-B). In slow-start, cwnd starts at 1 and increases exponentially (increases by one for each ACK received). Once the first loss is detected, cwnd is halved and the sender transitions to congestion-avoidance, where cwnd increases linearly ( $1 /$ cwnd for each ACK received). Subsequent drops cause further halving of cwnd, without the sender ever leaving congestion-avoidance. An important but subtle point in halving cwnd is that when a packet is sent, not only a timestamp but the current value of cwnd is saved in the to-be-ack list. When a packet drop is detected, then cwnd is actually set equal to half the value of cwnd at the time the packet was sent and not half the current value of cwnd. This makes the algorithm fairly

\footnotetext{
${ }^{4}$ We currently have an implementation of TCP-PR in the Linux kernel. In order to compute $\alpha^{(1 /\lfloor\text { crnd }\rfloor)}$ in the kernel, we employ Newton's method through the following loop:

$1 x:=$ result from last calculation or 1 if there has not been a previous calculation of $\alpha^{(1 /\lfloor\text { crnd }\rfloor)}$

2 while $\mid x-((\lfloor$ cwnd $\rfloor-1) /\lfloor$ cwnd $\rfloor) x+\left(\alpha /\lfloor\right.$ cwnd $\left.\left.\rfloor x^{\lfloor\text {cwnd }\rfloor-1}\right)\right) \mid>(1-$ a) $10^{-3}$

$3 x:=((\lfloor$ cwnd $\rfloor-1) /\lfloor$ cwnd $\rfloor) x+\left(\alpha /\lfloor\right.$ cwnd $\left.\rfloor x^{\lfloor\text {crnd }\rfloor-1}\right)$

4 end

The larger the value of $n$, the better the approximation. In our implementation, we are using $n=10$. However, if cwnd is bounded by a small enough value, it might be simpler to save $\alpha^{(1 /\lfloor\text { crnd }\rfloor}$ in a look-up table.
}

insensitive to the delay between the time a drop occurs until it is detected.

To prevent bursts of drops from causing excessive decreases in cwnd, once a drop is detected a snapshot of the to-be-sent list is taken and saved into an auxiliary list called memorize. As packets are acknowledged or declared as dropped, they are removed from the memorize list so that this list contains only those packets that were sent before cwnd was halved and have not yet been unaccounted for. When a packet in this list is declared dropped, it does not cause cwnd to be halved. The rational for this is that the sender already reacted to the congestion that caused that burst of drops. This type of reasoning is also present in TCP-NewReno and TCP-SACK.

The pseudo-code in Table I corresponds to the algorithm just described. Table II summarizes the notation used in the code.

Remark 1: From a computational view-point, TCP-PR is more demanding than TCP-(New)Reno because it requires the sender to maintain the list to-be-ack of packets whose acknowledgment are pending, but is not significantly more demanding than TCP-SACK. It does maintain the extra memorize list used to detect drop bursts, but this list is empty most of the time and otherwise only needs to contain pointers to packets also in the to-be-ack list. Recall that the transport layer must maintain the data to be transmitted until the packet has been ACKed by a cumulative ACK. If static memory allocation is used, the transport layer must allocate enough memory to hold a maximum sender's window's worth of packets. In the typical case where the MSS is several hundred to over a thousand bytes, TCP-PR's requirement of two bytes per packet for timestamps and lists of pointers results in a relatively minor increase in the transport layer's memory requirements. On the other hand, $\alpha^{1 /\lfloor\text { crnd }\rfloor}$ must be computed every time \cwnd $\rfloor$ is incremented and requires a number of multiplications, divisions and additions. Alternatively, $\alpha^{1 /[\text { cwnd }\rfloor}$ can be tabulated requiring table look-up operations. Clearly, table look-ups are less computationally intensive, but require more memory (again, this memory demand is small compared to what is required for packet buffering).

\section{B. Extreme Losses}

When more than half of a window's worth of packets is dropped, TCP-NewReno/SACK may timeout in the fast-recovery mode [20]. This is because not enough ACKs are received for the congestion window to open and allow for the sender to perform the needed retransmissions. The occurrence of a timeout typically depends on the number of packets dropped, the congestion window size cwnd, the round-trip time, and the value of $R T O$. In the ns-2 simulations whose results we report, we observed timeouts in TCP SACK when more than cwnd $/ 2+1$ packets are dropped within a window, which is consistent with the results in [20]. TCP-NewReno and SACK also enter the timeout mode when the retransmitted packets are lost or when drops occur while the congestion window size is smaller than 4. In the latter case, there are not enough ACKs to trigger fast recovery, so a timeout eventually occurs.

The "correct" behavior of congestion control under extreme losses is somewhat controversial and perhaps the more reasonable approach is to leave to the application to decide what to do 
TABLE I

PSEUdo-CODE FOR TCP-PR (CF. NOTATION IN TABLE II)

\begin{tabular}{|c|c|c|}
\hline Event & & Code \\
\hline initialization & $\begin{array}{l}1 \\
2 \\
3 \\
4\end{array}$ & $\begin{array}{l}\text { mode }:=\text { slow-start } \\
\text { cwnd }:=1 \\
\text { ssthr }:=+\infty \\
\text { memorize }:=\emptyset\end{array}$ \\
\hline $\begin{array}{l}\text { time }>\text { time }(n)+\text { mxrtt } \\
(\text { drop detected for packet } n \text { ) }\end{array}$ & $\begin{array}{r}5 \\
6 \\
7 \\
8 \\
9 \\
10 \\
11 \\
12 \\
13\end{array}$ & $\begin{array}{l}\text { remove }(\text { to-be-ack, } n) \\
\text { add(to-be-sent, } n) \\
\text { if not is-in(memorize, } n) \text { then } / * \text { new drop */ } \\
\quad \text { memorize }:=\text { to-be-ack } \\
\quad \text { cwnd }:=\text { cwnd }(n) / 2 \\
\quad \text { ssthr }:=\text { cwnd } \\
\text { else } / * \text { other drops in burst */ } \\
\text { remove(memorize, } n \text { ) } \\
\text { flush-cwnd () }\end{array}$ \\
\hline ack received for packet $n$ & $\begin{array}{l}14 \\
15 \\
16 \\
17 \\
18 \\
19 \\
20 \\
21 \\
22 \\
23\end{array}$ & $\begin{array}{l}\text { srtt }=\max \left\{\alpha \frac{1}{\text { cwnd }} \times \text { srtt, time }- \text { time }(n)\right\} \\
\text { mxrtt }:=\beta \times \text { srtt } \\
\text { remove }(\text { to }- \text { be }- \text { ack, } n) \\
\text { remove }(\text { memorize, } n) \\
\text { if mode = slow-start and cwnd }+1 \leq \text { ssthr then } \\
\text { else } \quad \text { cwnd }:=\text { cwnd }+1 \\
\text { mode }:=\text { congestion-avoidance } \\
\text { cwnd }:=\text { cwnd }+1 / \text { cwnd } \\
\text { flush-cwnd () }\end{array}$ \\
\hline flush-cwnd() & $\begin{array}{l}24 \\
25 \\
26 \\
27 \\
28\end{array}$ & $\begin{array}{l}\text { while cwnd }>\mid \text { to-be-ack } \mid \text { do } \\
k=\text { send(to-be-sent }) \\
\text { remove }(\text { to-be-sent, } k) \\
\text { add (to-be-ack, } k) \\
\text { time }(k)=\text { time }\end{array}$ \\
\hline
\end{tabular}

TABLE II

NOTATION USED IN TABLES I AND III

\begin{tabular}{ll}
\hline time & current time \\
time $(n)$ & time at which time packet $n$ was sent \\
cwnd $(n)$ & congestion window at the time packet $n$ was sent \\
is-in(list, $k)$ & returns true if the packet $k$ is in the list list \\
add(list, $k)$ & add the packet $k$ to the list list \\
remove(list, $k)$ & remove the packet $k$ from list list (if $k$ is not in list do nothing) \\
$\mid$ ist $\mid$ & number of elements in the list list \\
$k=$ send(list) & send the packet in list list with smallest seq. number, returning the seq. number \\
\hline
\end{tabular}

in this case. However, we have found that without special attention to the behavior during extreme losses, TCP-PR is unfair to today's implementations of TCP. In order to maintain fairness (a key goal of this work), we propose a version of TCP-PR that mimics TCP-SACK's timeout, i.e., upon detecting an extreme loss situation, TCP-PR sets SSTHRESH to cwnd/2, resets cwnd to one, performs exponential back-off, etc. It should be emphasized that this variation of TCP-PR is not required for the proper functioning of TCP-PR in sense that throughput is maintained without this enhancement. Rather, this variation results in lower, but more fair, throughput in high loss situations.

TCP-PR detects extreme losses by counting the number of packets lost in a burst. This can be done using a counter cburst that is incremented each time a packet is removed from the memorize list due to drops and is reset to zero when this list becomes empty. We recall that this list is usually kept empty but when a drop occurs it "memorizes" the packets that were outstanding. In the spirit of TCP-NewReno and TCP-SACK, packets from this list that are declared dropped do not lead to further halving of the congestion window.

To emulate as close as possible what happens during a TCPNewReno or TCP-SACK timeout, we check if cburst (and therefore the number of drops in a burst) exceeds cwnd $/ 2+1$, or if a drop is detected while cwnd $<4$, or if a retransmitted packet is dropped. When one of these conditions occurs, we reset $\mathrm{cwnd}=1$ and transition to the slow-start mode. Moreover, and for fairness with implementations of TCP-NewReno/SACK that use coarse-grained timers, we increase mxrtt to one second and delay sending packets by mxrtt [21]. If further (new) drops occur while cwnd $=1$, instead of dividing cwnd by two we double mxrtt, thus emulating the usual exponential back-off. The pseudo-code in Table III implements this algorithm. In this pseudo-code, we also inhibited increments of cwnd while the memorize list is not empty. This was also done to improve fairness with respect to TCP-NewReno/SACK, because it emulates the fact that in these algorithms cwnd only goes back to the usual increase of $1 /$ cwnd per ACK after the sender leaves the fast-recovery mode.

\section{SELECTION OF TCP-PR PARAMETERS}

In this section, we discuss the selection of the parameters $\alpha$ and $\beta$ used in the estimation of the maximum round-trip time mxrtt. When the time elapsed since a packet was sent exceeds mxrtt and its acknowledgment has not yet arrived, TCP-PR assumes the packet was dropped and divides the congestion window by two. However, there is the risk that if mxrtt is set too low, the algorithm will assume that a packet has been lost when it merely experienced a large round-trip time. We refer to such events as spurious timeouts. While occasional spurious timeouts are of little consequence, throughput may suffer severely if they occur too frequently.

In order to determine adequate values for $\alpha$ and $\beta$ that reduce the occurrence of spurious timeouts, we employ two different methods: 1) an analytical approach that determines a worstcase relationship between the rate of spurious timeouts and the 
mxrtt will not decrease and further spurious timeouts will not occur. As discussed in Section III, after $K$ congestion windows worth of packets have been ACKed, srtt $=\alpha^{K} \times R T T_{\max }$. Therefore, a spurious timeout will be produced by a packet taking the long route $K \times R T T_{\min }$ seconds after the previous one, as long as

$$
\beta \times \operatorname{srtt}=\beta \times \alpha^{K} \times R T T_{\max }<R T T_{\max } .
$$

We conclude from here that the minimum number of shorter round-trip times between spurious timeouts is equal to $K=$ $\log \beta /-\log \alpha$. In this worst-case situation, most packets take the shorter path and therefore the average $R T T$ is essentially $R T T_{\min }$. We can summarize our conclusions as follows. When (3) does not hold there will be no spurious timeouts, otherwise the time between spurious timeouts must be larger than

$$
\frac{\log \beta}{-\log \alpha} \times \overline{R T T}
$$

where $\overline{R T T}$ is the average round-trip time. This means that $\beta=3$ and $\alpha=0.999$ result in low rates for spurious timeouts (approximately $1000 \times \overline{R T T}$ seconds between spurious timeouts) and yield good performance, as confirmed in the following sections.

Remark 2: This worst-case analysis also applies to conditions such as highly variable processing delay within a router, or delay variations due to link layer ARQ or media access in wireless channels which may also cause spurious timeouts. Similarly to the scenario investigated above, the worst case situation is when the latency mostly takes a small value and occasionally jumps to a large value.

\section{B. Analysis of RTT Traces}

The above analysis determined the worst-case frequency of spurious timeouts. RTT traces can be utilized to estimate the probability of getting a spurious timeout in the more typical case of today's Internet. Using a July 25, 2001 snapshot of round-trip times from the National Laboratory for Applied Network Research (NLANR) data set, we computed empirical probability of spurious timeouts. The total data set consists of nearly 13000 connections between 122 sites and 17.5 million round-trip time measurements. This data consisted of time series of round-trip times for each connection with each time series containing 1440 round-trip times (one sample per minute over the entire day). For each time series, the srtt and mxrtt were computed and spurious timeouts noted. This process was repeated for several values of $\alpha$ and $\beta$. Fig. 2 shows the probability of a drop versus the parameters $\alpha$ and $\beta$.

For these computations, it was assumed that cwnd $=1$. However, for cwnd $>1$ we have that $\alpha<\alpha^{1 / \text { crnd }}$ and therefore

$$
\begin{aligned}
\operatorname{mxrtt}_{k+1} & =\beta \times \operatorname{srtt}_{k+1} \\
& =\beta \times \max \left\{\alpha^{\frac{1}{\text { cmd }}} \times \operatorname{srtt}_{k}, \text { sampleRTT }\right\} \\
& \geq \beta \times \max \left\{\alpha^{1} \times \operatorname{srtt}_{k}, \text { sampleRTT }\right\} .
\end{aligned}
$$

Hence, assuming cwnd $=1$ actually reduces mxrtt, leading to an overestimate on the number of spurious timeouts. The

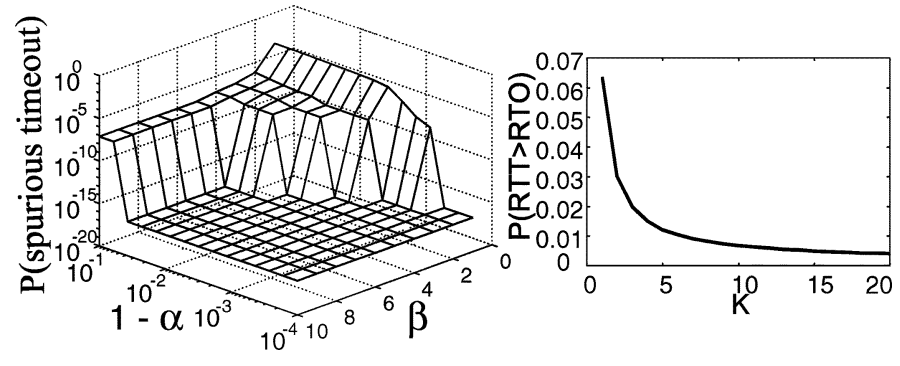

Fig. 2. Right: probability of spurious timeouts computed from the NLANR data set. For many pairs of $\alpha, \beta$ there were no spurious timeouts. However, in order to view the data on a log scale a perturbation of $10^{-16}$ has added. Hence, all the pairs $\alpha, \beta$ that show a probability of $10^{-16}$ actually had no spurious timeouts at all. Left: probability of spurious timeouts when Van Jacobson's Algorithm is used.

data used was also collected at one minute intervals, whereas TCP-PR would likely sample $R T T$ much more frequently. Since $R T T$ is positively correlated [22], large jumps are observed less frequently when $R T T$ is sampled at closely spaced intervals. The more frequent sampling that occurs within TCP-PR would likely drive down the probability of spurious timeouts even further.

Despite these two conservative assumptions, Fig. 2 shows that as long as $\beta>1$ and $\alpha>0.99$ the probability of a spurious timeout occurring is less than $10^{-7}$. For $\alpha>0.999$ and $\beta \geq$ 2 , the probability of a spurious timeout becomes vanishingly small.

Standard implementations of TCP [23] compute $R T O$ which is a filtered version of $R T T$ that is used to trigger TCP's timeout. Typically, $R T O$ is computed using Van Jacobson's algorithm [24]

$$
\begin{aligned}
\operatorname{srtt}_{k+1} & =\frac{7}{8} \operatorname{srtt}_{k}+\frac{1}{8} R T T_{k} \\
\operatorname{DevRTT}_{k+1} & =\frac{3}{4} \operatorname{DevRTT}_{k}+\frac{1}{4}\left|s r t t_{k}-R T T_{k}\right| \\
R T O_{k} & =\max \left(\operatorname{srtt}_{k}+K \times \operatorname{DevRTT_{k},MinRTO}\right) .
\end{aligned}
$$

While the typical value of $K$ is 4 , there is some discrepancy over the value of MinRTO. Often, it is assumed that $\operatorname{MinRTO}=1$ second (as specified in RFC 2988 [21]), but some implementations use different values, e.g., BSD and MS Windows uses $500 \mathrm{~ms}$, and Linux uses $200 \mathrm{~ms}$. Fig. 2 shows the empirical probability of $R T T>R T O$ from the NLARN data set under the assumption that MinRTO $=0$. For $K=4$, we obtained $P(R T T>R T O) \approx 0.0124$, i.e., a little more than $1 \%$ of all packets would timeout. It is difficult to determine the best value of MinRTO. If RTT > MinRTO, then we can expect $1 \%$ of packets sent will trigger a spurious timeout. For RTO too large, the throughput of TCP-PR would be reduced. While it is possible that an intermediate value of MinRTO would result in less than $1 \%$ spurious timeouts while maintaining high throughput, it is difficult to find a single MinRTO that works well in many situations. A similar conclusion was also reached in [25] where no obvious "sweet spots" were found for MinRTO when used in TCP. 


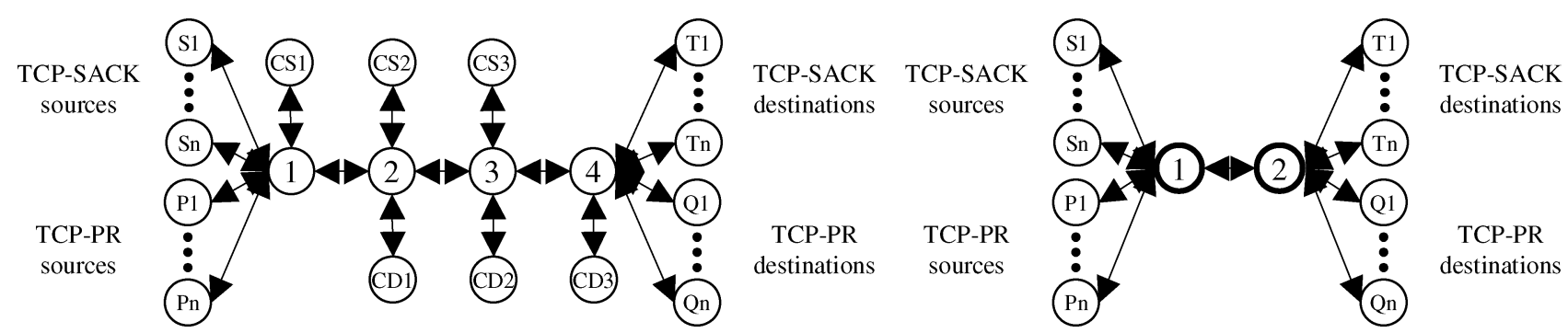

Fig. 3. Left: parking-lot topology with multiple bottlenecks and cross traffic. The source and destination are labeled $\mathrm{S}$ and $\mathrm{D}$ respectively. The cross-traffic connections are CS1 $\rightarrow$ CD1, CS1 $\rightarrow$ CD2, CS1 $\rightarrow$ CD3, CS2 $\rightarrow$ CD2, CS2 $\rightarrow$ CD3, and CS3 $\rightarrow$ CD3. The data rates are: $5 \mathrm{Mb} / \mathrm{s}$ for CS1 $\rightarrow 1,1.66$ $\mathrm{Mb} / \mathrm{s}$ for CS2 $\rightarrow 2,2.5 \mathrm{Mb} / \mathrm{s}$ for CS3 $\rightarrow 3$, and $15 \mathrm{Mb} / \mathrm{s}$ for all other links. This results in the following three bottlenecks: $1 \rightarrow 2,2 \rightarrow 3$, and $3 \rightarrow 4$. Right: the dumbbell topology.

\section{Performance AND FAirness Without PACKET REORDERING}

Two issues arise when considering TCP-PR over networks without packet reordering: performance and fairness. The first issue is whether TCP-PR performs as well as other TCP implementations under "normal" conditions, i.e., no packet reordering. Specifically, for a fixed topology and background traffic, does TCP-PR achieve similar throughput as standard TCP implementations? The second concern is whether TCP-PR and standard TCP implementations are able to coexist fairly. To some extent, the fairness issue encompasses the performance issue: if TCP-PR competes fairly against standard TCP implementations in a variety of network conditions, then it seems reasonable that TCP-PR and other TCP implementations are able to achieve similar throughput (and thus perform similarly) when exposed to similar network conditions. Therefore, while this section focuses on fairness, it indirectly addresses the performance issue. Additionally, in Section VI, we also show that, when no packet reordering occurs, TCP-PR achieves the same throughput as other TCP implementations.

We performed extensive ns-2 [26] simulations to show that TCP-PR is fair with respect to standard TCP implementations, for a wide range of network conditions and topologies. In this section, a sample of our simulation results is presented, with attention focused on the compatibility with TCP-SACK [27]. One of the topologies we use is the dumbbell topology, also known as single-bottleneck. A number of simulation-based studies have used the dumbbell topology to evaluate the performance of network protocols. One recent example is the comparison between the performance (including fairness) of TCP-SACK and an implementation of the "TCP-friendly" formula [28]. The other topology used is the parking-lot topology, which includes multiple bottleneck links and has also been employed in a number of recent performance studies of network protocols including [29] and [30]. Fig. 3 shows the parking-lot and dumbbell topologies used, including the source and destination nodes for the cross traffic. Previous studies that used the parking-lot topology only included cross traffic between nodes $\mathrm{CS} 1 \rightarrow \mathrm{CD} 1$, CS2 $\rightarrow \mathrm{CD} 2$, and $\mathrm{CS} 3 \rightarrow \mathrm{CD} 3$. In our simulations, we also considered cross traffic between CS1 $\rightarrow$ CD2, $\mathrm{CS} 1 \rightarrow \mathrm{CD} 3$, and CS2 $\rightarrow$ CD3. For the single-bottleneck (dumbbell) topology, we ran simulations both with and without HTTP background traffic. When background traffic was used, it corresponded to around $10 \%$ of the total traffic. ${ }^{5}$ HTTP traffic flowed from node 1 to node 2; more specifically, we set up five Web server-client pairs, each of which running ten concurrent connections. All Web clients ran in node 2 and all Web servers in node 1 so that the HTTP traffic direction coincides with the TCP flows under study. For the parking-lot topology, we always considered HTTP cross traffic. Eight Web server-client pairs were configured each of which with ten concurrent active sessions. While CS1 $\rightarrow$ CD1 and CS3 $\rightarrow$ CD3 have two pairs of Web server-client each, all other pairs have a single Web server-client pair. In both topologies, each Web session used the following parameters: inter-page time was exponentially distributed with mean 1 second, the number of objects per page was uniformly distributed with mean 1 , the inter-object time was exponentially distributed with mean $10 \mathrm{~ms}$, and object size was Pareto-distributed with mean $40 \mathrm{~KB}$ and shape parameter equal to $1.2 .{ }^{6} \mathrm{~A}$ large number of distinct link speeds and number of flows were investigated.

Following the approach taken in [28], the fairness of TCP-PR to TCP-SACK is judged by simulating an equal number of TCP-PR and TCP-SACK flows. These flows have a common source and destination. The steady state fairness can be quantified with a single number, the mean normalized throughput. If there are $n$ flows, then the normalized throughput of flow $i$ is

$$
T_{i}=\frac{x_{i}}{\frac{1}{n} \sum_{j=1}^{n} x_{j}}
$$

where the throughput, $x_{i}$, is the total data sent during the last 60 seconds of the simulation. The mean normalized throughput for a particular protocol is the average value of $T_{i}$, averaged over all the flows of that protocol. Note that if $T_{i}=1$, then flow $i$ achieves the average throughput. Similarly, if the mean normalized throughout of both protocols is one, then they achieved the same average throughput.

Figs. 4-10 show the normalized throughput and the mean normalized throughput for different numbers of flows, topologies, link speeds, queue disciplines, propagation delays, and with and

\footnotetext{
${ }^{5}$ Generating HTTP background traffic to correspond to $10 \%$ of the total traffic was motivated by the observation that, "while the exact fraction of short-lived traffic found on the Internet is unknown, it appears that short-lived flows make up for at least $10 \%$ of the total Internet traffic" [31].

${ }^{6}$ These are the same parameters used by sample ns- 2 scripts included in the ns-2 distribution; furthermore, these same parameters have also been used in simultaions conducted by other researchers (e.g., [32]).
} 

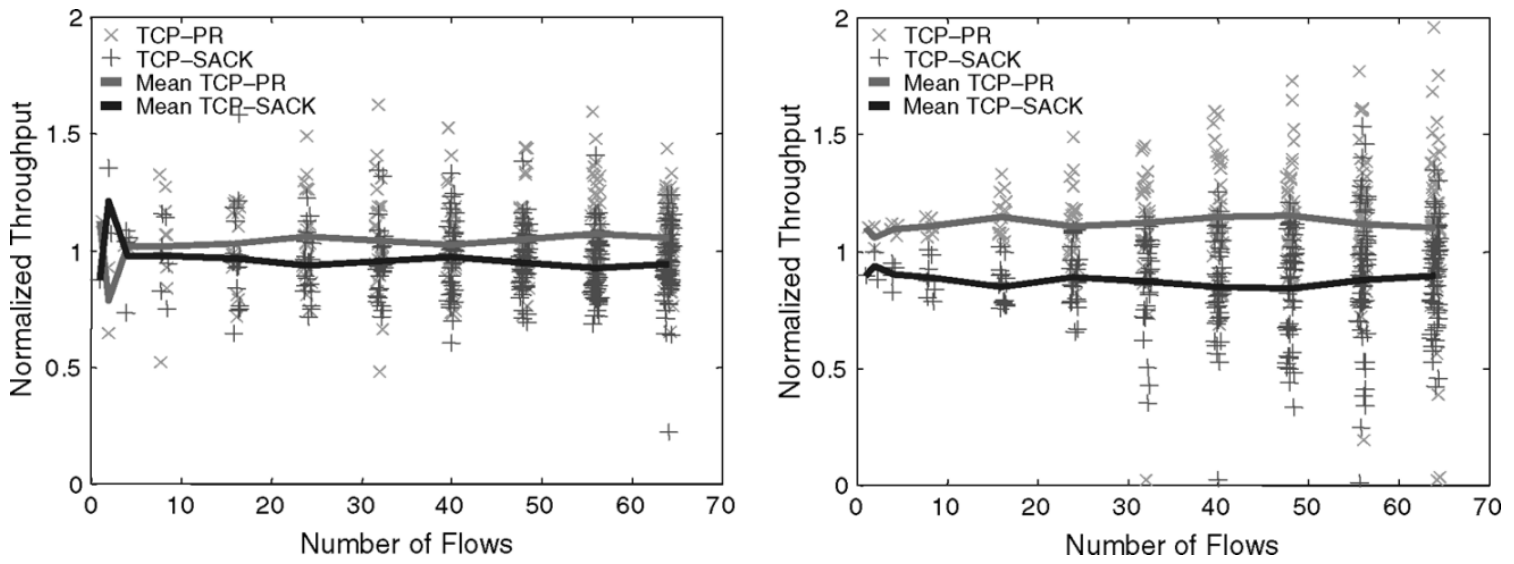

Fig. 4. TCP-PR and TCP-SACK Normalized Throughput over a Single Bottleneck Topology with RED Queue Discipline. The left-hand figure shows the case of $200 \mathrm{~ms}$ round-trip propagation delay and a 250 packet queue, while the right-hand plot shows the case of 20 ms round-trip propagation delay and a 25 packet queue. In both cases, the bottleneck link bandwidth was $15 \mathrm{Mb} / \mathrm{s}$.
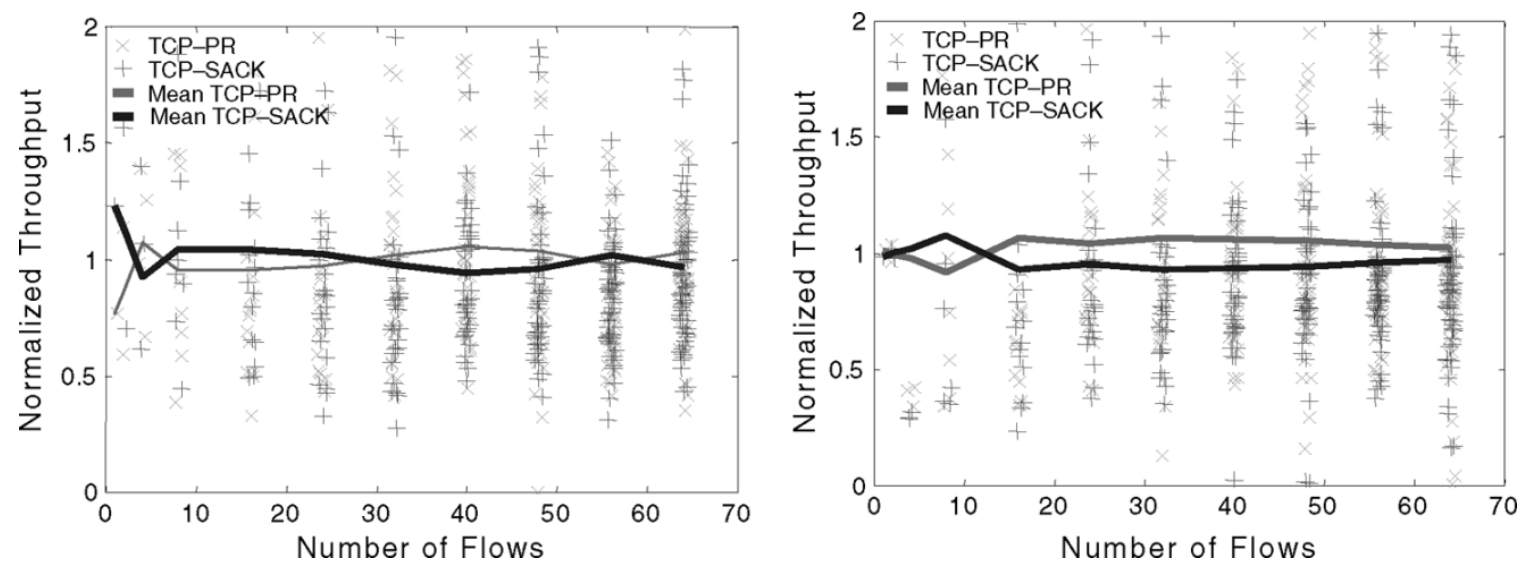

Fig. 5. TCP-PR and TCP-SACK Normalized Throughput over a Single Bottleneck Topology with Drop-Tail Queue Discipline. The left-hand figure shows the case of $200 \mathrm{~ms}$ round-trip propagation delay and a 250 packet queue, while the right-hand plot shows the case of 20 ms round-trip propagation delay and a 250 packet queue. In both of these cases, the bottleneck link bandwidth was $15 \mathrm{Mb} / \mathrm{s}$.
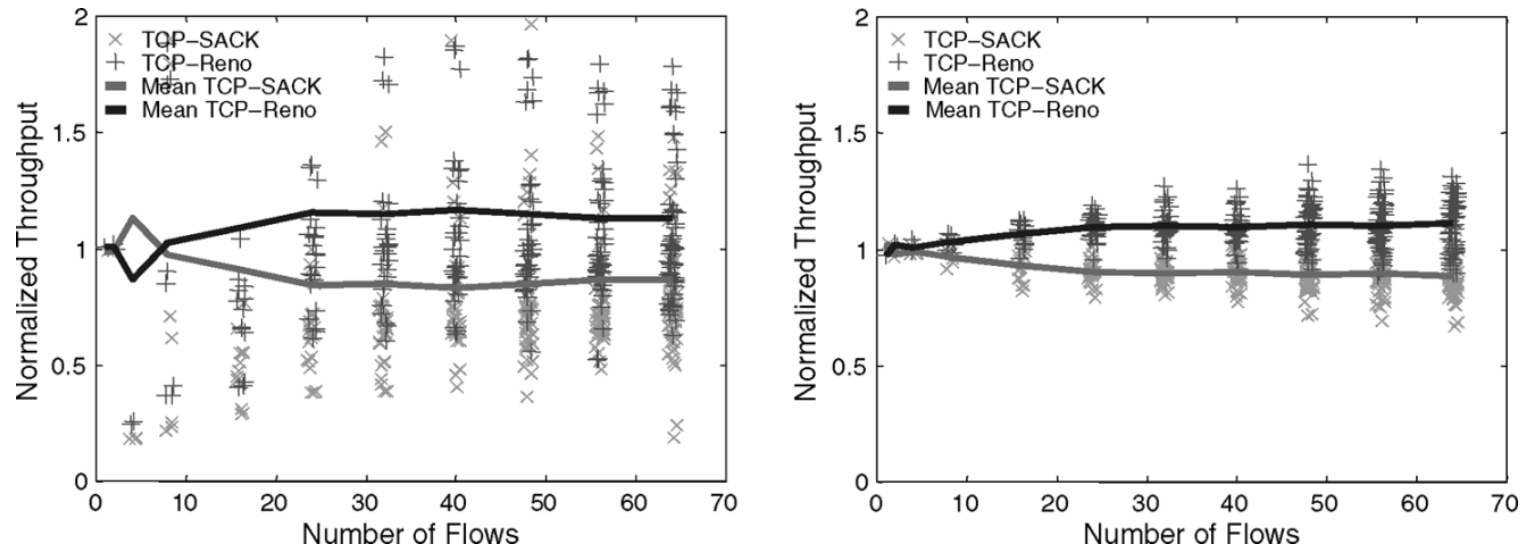

Fig. 6. Normalized Throughput of TCP-Reno and TCP-SACK. The left-hand plot shows the throughput of TCP-SACK and TCP-Reno for the drop-tail queue discipline, while the right-hand plot shows the results for RED queue discipline. In these simulations the topology was a single bottleneck topology with a $15 \mathrm{Mb} / \mathrm{s}$ bandwidth bottleneck. The round-trip propagation delay was $20 \mathrm{~ms}$ and the queue size was 250 packets.

without cross traffic. In these experiments, $\alpha$ and $\beta$ were fixed at 0.999 and 3.0, respectively. For comparison purposes, Figs. 6 and 8 compare the throughput when TCP-SACK and TCP-Reno compete for bandwidth. From the graphs, it is clear that the two versions of TCP-PR and TCP-SACK compete fairly over a wide range of traffic conditions and thus exhibit similar performance. 

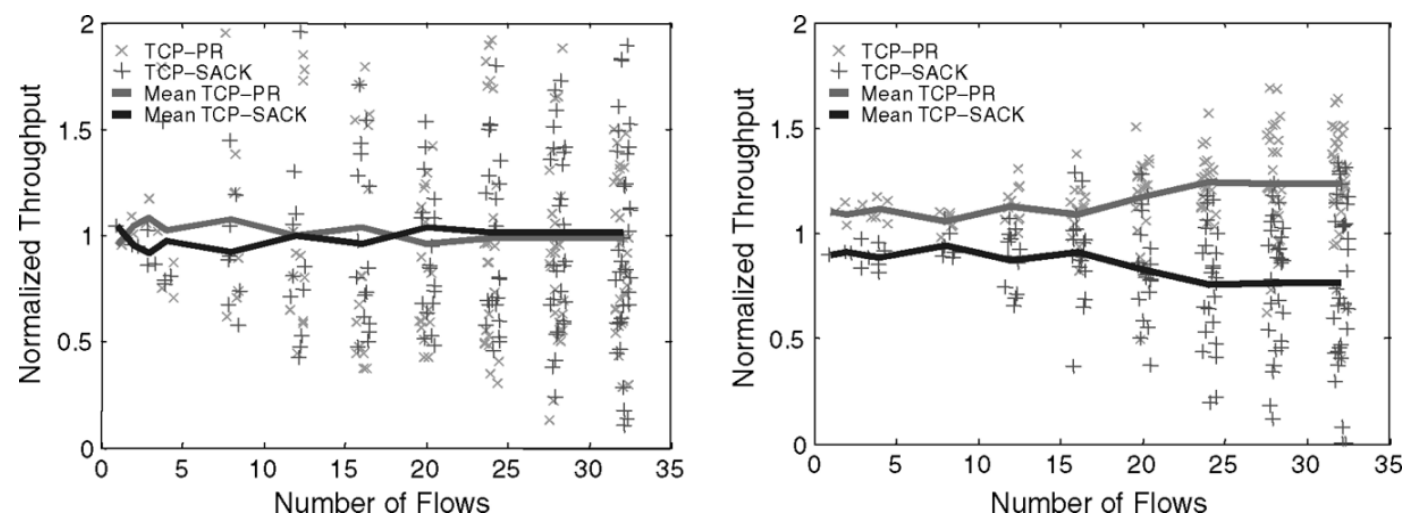

Fig. 7. Normalized Throughput for TCP-PR and TCP-SACK. The left-hand plot shows the results for the drop-tail queue discipline while the right-hand plot shows the results for RED queue discipline. In these simulations the topology was a single bottleneck topology with a $1.5 \mathrm{Mb} / \mathrm{s}$ bandwidth bottleneck. The round-trip propagation delay was $20 \mathrm{~ms}$ and the queue size was 25 packets.

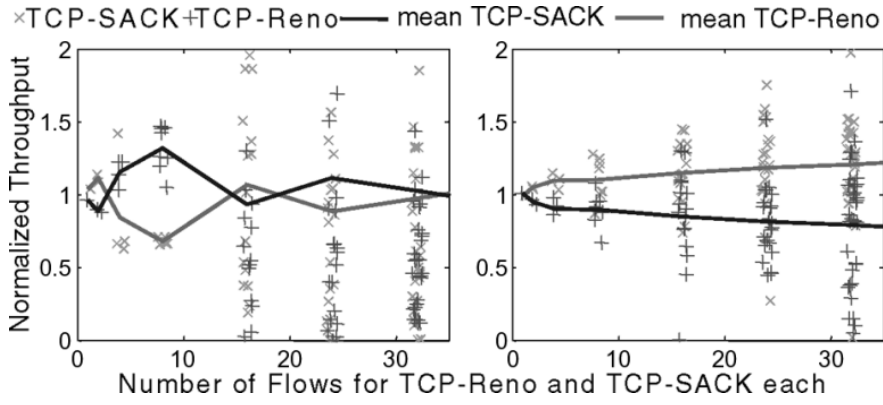

Fig. 8. Normalized throughput of TCP-Reno and TCP-SACK. The left-hand plot shows the throughput of TCP-SACK and TCP-Reno for the drop-tail queue discipline, while the right-hand plot shows the results for RED queue discipline. In these simulations the topology was a single bottleneck topology with a $1.5 \mathrm{Mb} / \mathrm{s}$ bandwidth bottleneck. The round-trip propagation delay was $20 \mathrm{~ms}$ and the queue size was 25 packets.

While the mean normalized throughput describes the average behavior of all flows, the coefficient of variation describes the variation of the throughput and is defined by

$$
C o V:=\frac{1}{\sum_{i \in I} T_{i}} \sqrt{\sum_{i \in I}\left(T_{i}-\frac{1}{|I|} \sum_{i \in I} T_{i}\right)^{2}}
$$

where $I$ denotes the set of flows of a particular protocol, and $|I|$ the number of elements in the set $I$. Fig. 11 shows the coefficient of variation for ten simulations as well as the mean coefficient of variation for the simulation set. From Figs. 4-11, we conclude that the mean and variance of the throughput for TCP-PR and TCP-SACK are similar. In light of these results, incremental TCP-PR deployment should have no adverse effects on competing flows that use other implementations of TCP.

Fig. 12 shows TCP-SACK's mean normalized throughput for different values of $\alpha$ and $\beta$. For these simulations, the number of flows was held constant at 64 total flows (32 TCP-SACK and 32 TCP-PR flows). Surprisingly, fairness is maintained for a wide range of $\alpha$ and $\beta$. Note that for $\beta=1$, TCP-SACK exhibits higher throughput. However, for $\beta$ larger than 1, both implementations achieve nearly identical performance. A large number of simulations show that these results are consistent for different levels of background traffic and different topologies. We noticed that even in situations where cross traffic causes extreme loss conditions (over 15\% drop probability), TCP-SACK only gets up to $20 \%$ more throughput when $\beta=10$, while throughput is essentially the same for $1<\beta<5$. Such extreme loss scenarios are not of particular concern since TCP's throughput is very low when the loss probability is this large.

Under normal traffic conditions, fairness is not so much evidence of the remarkableness of TCP-PR, but rather it attests to the robustness of additive-increase/multiplicative-decrease (AIMD) schemes. An important feature of these schemes is that if two flows detect drops at the same rate, then their congestion windows will converge to the same value. In fact, it was shown in [33] and, in more detail, in [34] that, at least for a dumbbell topology, competing TCP flows converge to the same bandwidth exponentially fast. While these proofs rely on the protocols being identical, they also point to the inherent stability of the AIMD scheme which is witnessed in the simulation results presented here.

While the focus here is on fairness, packet delay is also of interest. TCP-PR results in delays that, on average, are similar to today's implementations of TCP, even though their loss detection mechanisms differ. In the case of a single packet loss in the middle of a file transfer, today's implementations of TCP will deliver the lost packet $(\beta-1) R T T$ s sooner than TCP-PR. We note that our simulations use $\beta=3$. On the other hand, if there are over $\beta$ losses, TCP-PR will deliver packets sooner than TCP New Reno. In the multiple loss scenario, the packet delay of TCP-SACK compared to TCP-PR depends (in a complicated way) on the total number of losses and the window size. In general, as the number of losses increases, the difference between TCP-PR and TCP-SACK decreases.

In the case of small file transfers, it is likely that a packet loss will not invoke triple duplicate ACKs (either because cwnd is small, or because the lost packet is near the end of the file). In the case that the packet is at the end of the file, TCP-PR will deliver the packet sooner than current TCP implementations. On the other hand, if the packet loss occurs when cwnd is small, TCP-PR will recognize the loss sooner than many of today's TCP implementations. Exceptions are implementations that use Limited Transmit [35], which allows packets to be sent even 

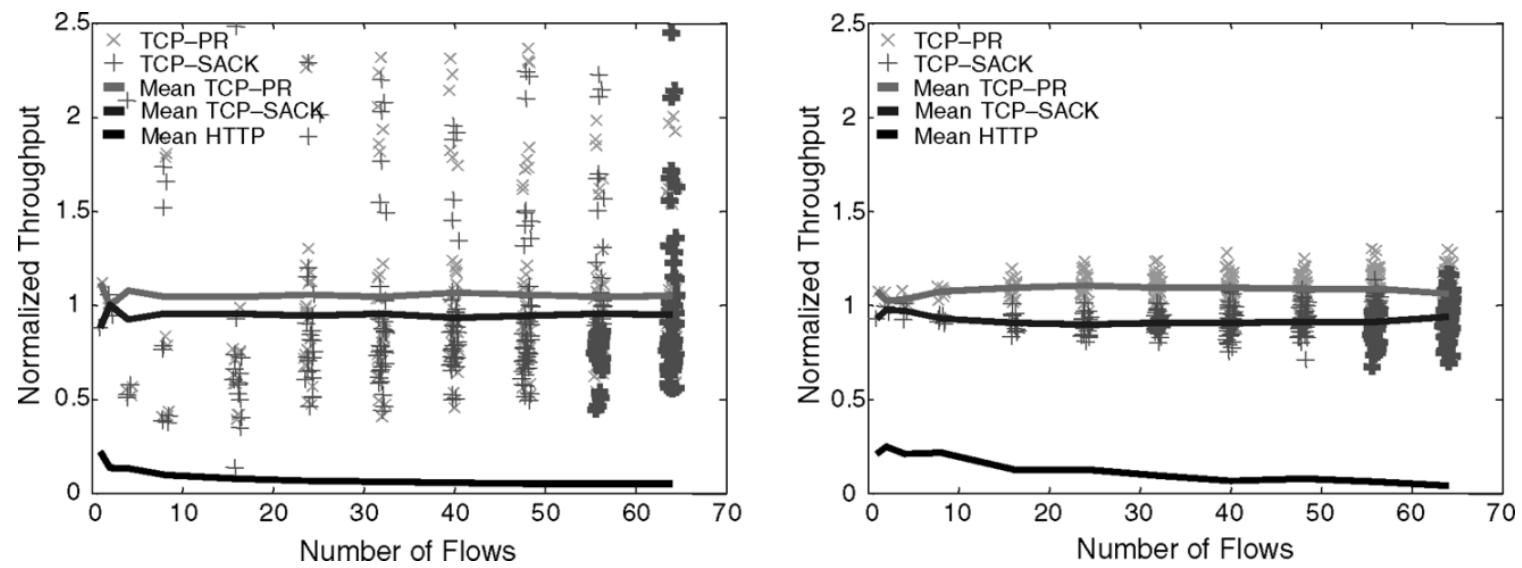

Fig. 9. Normalized throughput for TCP-PR and TCP-SACK with HTTP Background Traffic. The left-hand plot shows the results for the drop-tail queue discipline while the right-hand plot shows the results for RED queue discipline. In these simulations the topology was a single bottleneck topology with a $15 \mathrm{Mb} / \mathrm{s}$ bandwidth bottleneck. The round-trip propagation delay of $20 \mathrm{~ms}$ and a queue size of 250 packets.

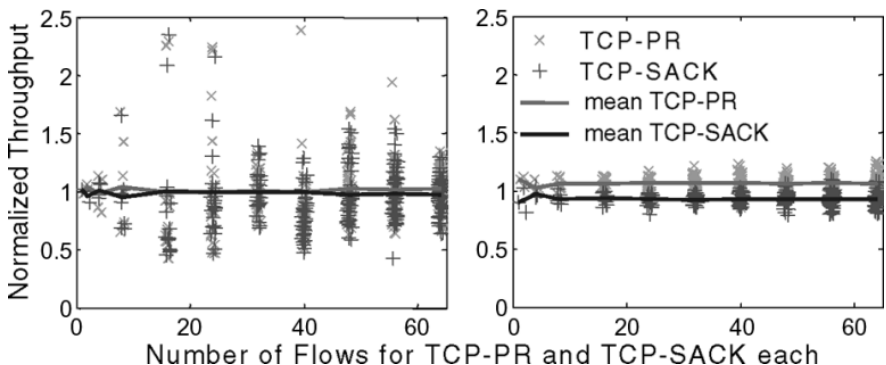

Fig. 10. Normalized Throughput for TCP-PR and TCP-SACK with HTTP Cross Traffic. The left-hand plot shows the results for the drop-tail queue discipline while the right-hand plot shows the results for RED queue discipline. In these simulations the topology was the parking-lot topology with a $15 \mathrm{Mb} / \mathrm{s}$ links. Each link has the round-trip propagation delay of $20 \mathrm{~ms}$ with a queue size of 250 packets.
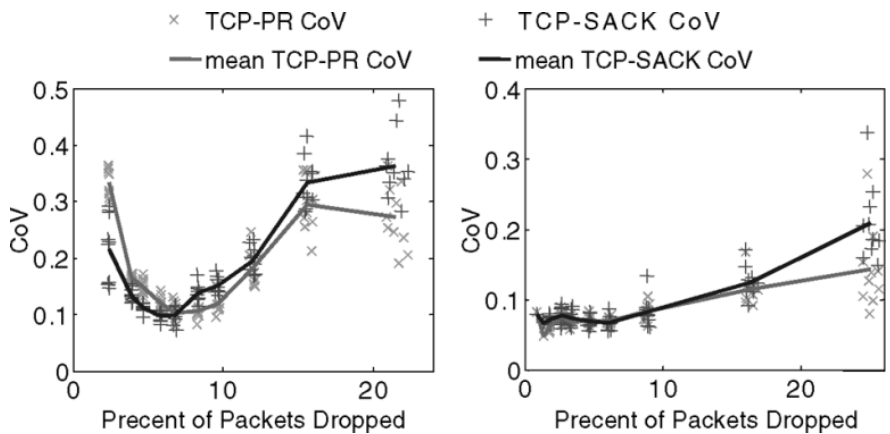

Fig. 11. Coefficient of variation. The coefficient of variation as a function of packet loss probability. The variation in loss probability was simulated by decreasing the link bandwidth. The left plot is the coefficient of variation for the dumbbell topology and the right plot is for the parking lot topology. The single bottleneck had a round-trip propagation delay of $20 \mathrm{~ms}$ and a queue size of 250 packets. The parking-lot topology had a round-trip propagation delay of $20 \mathrm{~ms}$ with a queue size of 250 packets. In both cases, the link speed was $15 \mathrm{Mb} / \mathrm{s}$ and the drop-tail queueing discipline was used. The parking-lot topology had HTTP cross traffic.

when DUP-ACKs arrive. Such implementations would detect the loss two RTTs after the lost packet is sent, whereas TCP-PR would detect it $\beta \times R T T$ after it was sent. Implementations that do not use Limited Transmit enter timeout in such situations. In order to maintain fairness with implementations that
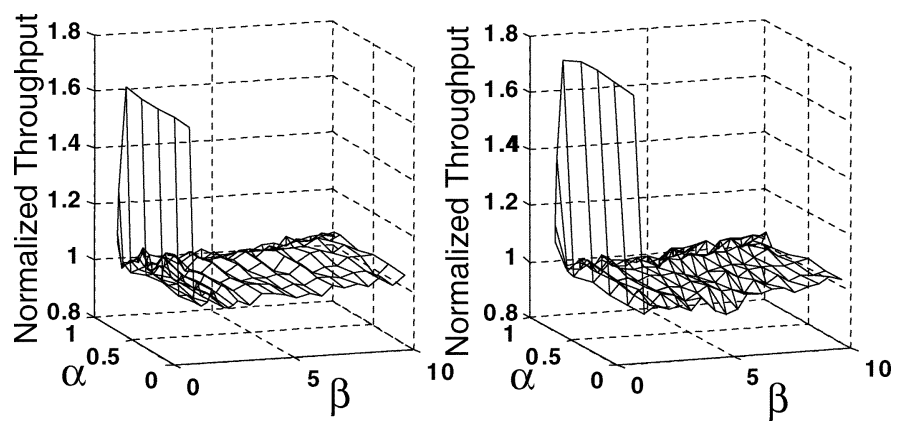

Fig. 12. TCP-SACK normalized throughput for different TCP-PR parameters. The left plot shows the mean normalized throughput of TCP-SACK using the single bottleneck topology, while the right plot shows normalized throughput for the parking-lot topology. The single bottleneck had a round-trip propagation delay of $20 \mathrm{~ms}$ and a queue size of 250 packets. The parking-lot topology had a two-way propagation delay of $20 \mathrm{~ms}$ with a queue size of 250 packets. In all cases, the link speed was $15 \mathrm{Mb} / \mathrm{s}$ and the drop-tail queueing discipline was used. The parking-lot topology had HTTP cross traffic.

do not use Limited Transmit, TCP-PR will enter the extreme loss state and will deliver the packet at the same time as these implementations.

\section{Vi. Performance Under Packet Reordering: COMPARISON WITH OTHER METHODS}

This section compares the performance of TCP-PR against existing algorithms that make TCP more robust to packet reordering. Two types of packet reordering are investigated: packet reordering due to queue swaps which might occur within a switch as suggested by [1], and reordering due to multipath routing. Our goal in designing TCP-PR is to provide a transport protocol that is suitable for environments that exhibit persistent reordering, yet achieving adequate performance (including fairness) in environments with no or occasional reordering. While queue swaps may lead to the latter scenarios, as discussed in Section IV, multipath routing typically results in persistent packet reordering and can be especially demanding since packets sent back-to-back may experience very different latencies.

Besides TCP-PR, several other approaches to TCP are considered. These include TCP-SACK with the DSACK feature 

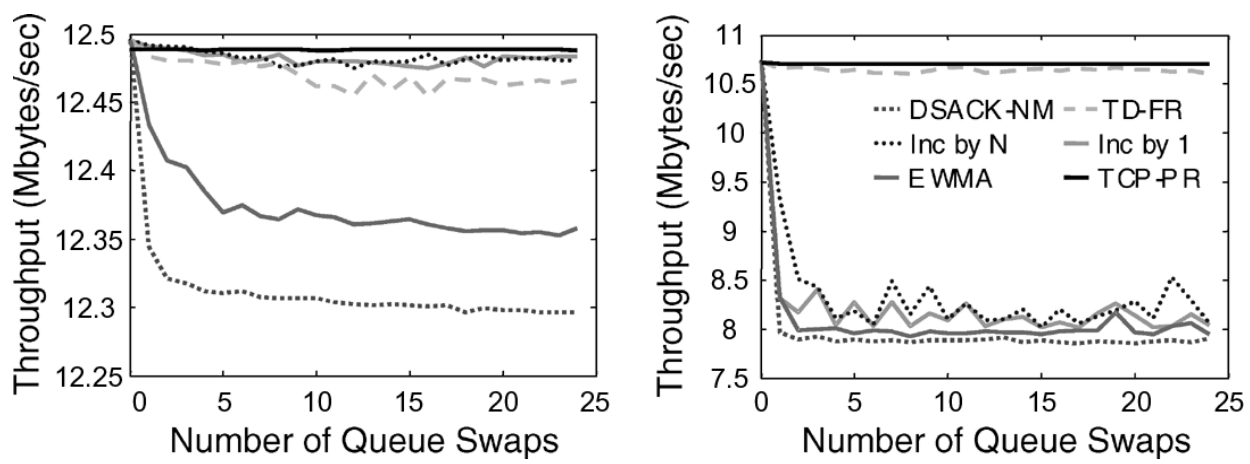

Fig. 13. Throughput as a function of the number of packet swaps per swap event. The left plot shows the throughput for a $30 \mathrm{~ms}$ propagation delay and the right plot for a $180 \mathrm{~ms}$ propagation delay.

enabled. In this case, spurious drops are detected, but no mitigation is performed. This method is labeled DSACK-NM. In [1], several methods were examined and are considered here as well. These methods use "limited transmit" [35] so that packets are still sent when duplicate ACKs arrive. These methods also adjust dupthresh. In the graphs that follow, Inc by 1 refers to the approach that increments the dupthresh by one every time a spurious retransmission is detected. Upon detecting a spurious retransmission, the method labeled Inc by $N$ increases the dupthresh such that the just observed spurious retransmission would not have occurred. The method labeled EWMA varies the dupthresh according to an exponentially weighted moving average filter. Another method first suggested in [18] and further investigated in [1] is referred to as time-delayed fast-retransmit $(T D-F R)$. In this case, fast retransmit is only entered when a triple duplicate ACK is observed and a certain amount of time has passed since the packet was sent. Recently another method for adapting dupthresh has been suggested [17]. Since a simulation implementation of this method is not yet available, it was not included in this comparison.

\section{A. Performance Under Packet Reordering Due to Queue Swaps}

In [1] the different versions of TCP were compared by examining their performance in the face of queue swaps. A queue swap is when two packets in a queue are exchanged. We assume that queue swap events occur every one second, and each event consists of $K$ individual packet swaps. The ns-2 simulations presented refer to a single bottleneck topology with drop-tail queuing, a maximum queue size of 250 packets, and one flow. The experiments were repeated for different propagation delays. Since the critical concern is whether or not the throughput is affected by queue swaps, Fig. 13 shows the relationship between throughput and the number $K$ of packet swaps per queue swap event.

The left plot in Fig. 13 shows the throughput for a $30 \mathrm{~ms}$ propagation delay and the right plot for a $180 \mathrm{~ms}$ propagation delay. In the low propagation delay case, most algorithms work relatively well. Indeed, even TCP-SACK with no special mitigation for packet reordering achieves a throughput that is merely $1.5 \%$ smaller than the other algorithms. However, for higher propagation delays the situation is quite different. In the right plot of Fig. 13, we observe that most methods only achieve a

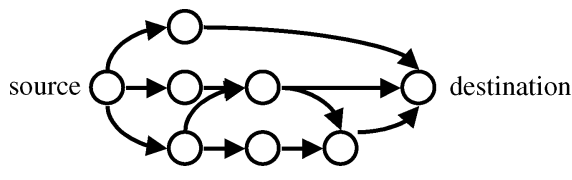

Fig. 14. Topology to compare TCP implemenations. Each link has a delay of $20 \mathrm{~ms}$, bandwidth of $10 \mathrm{Mb} / \mathrm{s}$, and queue has a size of 100 packets.

throughput that is $25 \%$ smaller than TCP-PR and TD-FR. In both cases the throughput achieved by TCP-PR is nearly independent of the number of packet swaps per swap event. This result is to be expected since reordering of packets in a queue will merely produce duplicate ACKs. The ACK arrival rate is not changed and hence TCP-PR's throughput is not affected.

\section{B. Performance Under Packet Reordering Due to Multipath Routing}

As before, we ran extensive simulations using ns-2 to compare the performance of the different algorithms in the face of persistent packet reordering due to multipath routing. The tests presented were performed on the topology shown in Fig. 14. Different sets of simulations were performed. In the first set, the propagation delay for each link was set to $10 \mathrm{~ms}$, while in the second set it was set to $60 \mathrm{~ms}$. These simulations were performed with and without background traffic.

Many multipath routing strategies are possible over this topology. We developed a family of strategies that is parameterized by a single variable $\varepsilon$ (cf. [36] for details). This parameter controls the degree to which routing accounts for link cost: When $\varepsilon=\infty$ the link cost is heavily penalized, resulting in minimum-hop routing. When $\varepsilon=0$ the link cost is not penalized at all and all independent paths from source to destination are used with equal probability. Intermediate values of $\varepsilon$ correspond to compromises between these two extreme cases. We compared the performance of TCP-PR with that of the various TCP versions with dupthresh compensation schemes in [1]. This was done for several fixed routing strategies, each corresponding to a distinct value of $\varepsilon$. In these simulations only one flow was active at a time.

Fig. 15 shows the throughput for various values of $\varepsilon$. The simulations show that for $\varepsilon=500$ (single-path routing), all methods achieve the same throughput. For $\varepsilon=0$ (full multi-path routing) most protocols other than TCP-PR suffer drastic decreases 


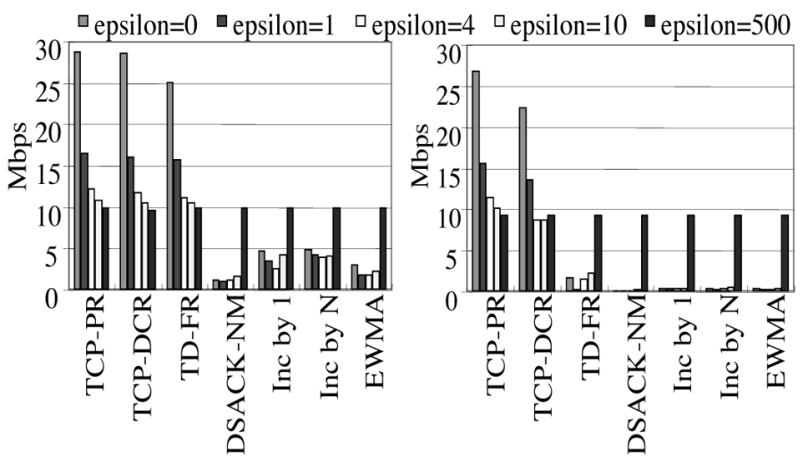

Fig. 15. Throughput for different TCP implementations and different degrees of multi-path routing. $\varepsilon=500$ corresponds to single path routing, whereas for smaller values of $\varepsilon$ alternative paths are sometimes used. In the limit $\varepsilon=$ 0 , all paths are used with equal probability. The left plot corresponds to the topology in Fig. 14 with a $10 \mathrm{~ms}$ propagation delay for each link and the right plot corresponds to the same topology but with a $60 \mathrm{~ms}$ propagation delay for each link.

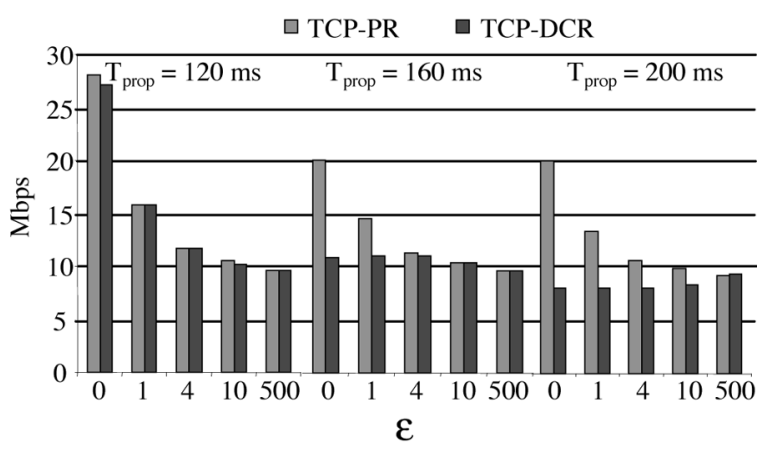

Fig. 16. Throughput of TCP-PR and TCP-DCR for different values of $\epsilon$ and different propagation delays.

in throughput. The exception is time-delayed fast-recovery (TD-FR) and TCP-DCR, which still achieves a reasonable throughput for small values of $\varepsilon$ when the propagation delay is small (the left plot in Fig. 15). However, as shown in Fig. 15 and Fig. 16, TD-FR and TCP-DCR still suffer a large decrease in throughput when the propagation delay is increased. The reason for this drop in throughput is that TD-FR and TCP-DCR make use of both dupthresh and timers. While the "limited transmit algorithm" attempts to reduce it, burstiness remains a problem for TD-FR over connections with long latency. These simulations demonstrate the effectiveness of TCP-PR's timer-based packet drop detection. This confirms that duplicate ACKs are indicative of packet loss in single path routing, but their occurrence convey little information when multi-path routing is utilized.

\section{CONCLUSION}

In this paper we proposed and evaluated the performance of TCP-PR, a variant of TCP that is specifically designed to handle persistent reordering of packets (both data and acknowledgment packets). Our simulation results show that TCP-PR is able to achieve high throughput when packets are reordered and yet is fair to standard TCP implementations, exhibiting similar performance when packets are delivered in order. From a computational view-point, TCP-PR is more demanding than TCP(New)Reno but carries essentially the same overhead as TCPSACK.
Because of its robustness to persistent packet reordering, TCP-PR allows mechanisms that introduce packet reordering as part of their normal operation to be deployed in the Internet. Such mechanisms include proposed enhancements to the original Internet architecture such as multi-path routing for increased throughput, load balancing, and security; protocols that provide differentiated services (e.g., DiffServ [8]); and traffic engineering approaches.

A Linux implementation of TCP-PR is under development and is available at [37]. Furthermore, TCP-PR is expected to work well in wireless multi-hop environments allowing wireless routing protocols to make use of multiple paths when available. While the protocol described in this paper focuses on wired networks, we plan to adapt it for wireless environments as part of our future work.

\section{REFERENCES}

[1] E. Blanton and M. Allman, "On making TCP more robust to packet reordering," ACM Comput. Commun. Rev., vol. 32, no. 1, 2002.

[2] V. Paxson, "End-to-end routing behavior in the Internet," in ACM SIGCOMM, 1996, pp. 25-38.

[3] J. Bennett and C. Partridge, "Packet reordering is not pathological network behavior," IEEE/ACM Trans. Netw., vol. 7, no. 6, pp. 789-798, Dec. 1999.

[4] L. Cottrell, "Packet reordering," [Online]. Available: http://www-iepm. slac.stanford.edu/monitoring/reorder/

[5] F. Wang and Y. Zhang, "Improving TCP performance over mobile ad-hoc networks with out-of-order detection and response," in $A C M$ MOBIHOC, 2002, pp. 217-225.

[6] T. Dyer and R. Boppana, "A comparison of TCP performance over three routing protocols for mobile ad hoc networks," in ACM MOBIHOC, 2001, pp. 56-66.

[7] G. Holland and N. Vaidya, "Analysis of TCP performance over mobile ad-hoc networks," in ACM MOBICOM, 1999, pp. 219-230.

[8] S. Blake, D. Black, M. Carlson, E. Davies, Z. Whang, and W. Weiss, "An architecture for differentiated services," RFC 2475, 1998.

[9] D. Bertsekas, Network Optimization: Continuous and Discrete Models. Belmont, MA: Athena Scientific, 1998.

[10] N. Taft-Plotkin, B. Bellur, and R. Ogier, "Quality-of-service routing using maximally disjoint paths," in Proc. IEEE/IFIP IWQoS'99, Jun. 1999, pp. 119-128.

[11] S. Bohacek, J. Hespanha, K. Obraczka, J. Lee, and C. Lim, "Secure stochastic routing," presented at the ICCCN'02, Miami, FL, 2002.

[12] R. Teixeira, K. Marzullo, S. Savage, and G. M. Voelker, "Characterizing and measuring path diversity of Internet topologies," presented at the ACM SIGMETRICS, San Diego, CA, Jun. 2003.

[13] A. Nasipuri and S. Das, "Demand multipath routing for mobile ad hoc networks," presented at the ICCCN'99, Boston, MA, Oct. 1999.

[14] M. Pearlman, Z. Haas, P. Sholander, and S. Tabrizi, "The impact of alternate path routing for load balancing in mobile ad hoc networks," presented at the ACM MobiHoc, Boston, MA, Aug. 2000.

[15] R. Ludwig and R. Katz, "The Eifel algorithm: making TCP robust against spurious retransmissions," ACM Comput. Commun. Rev., vol. 30 , no. 1,2000

[16] S. Floyd, J. Mahdavi, M. Mathis, and M. Podolsky, "An extension to the Selective Acknowledgment (SACK) option for TCP," RFC 2883, 2000.

[17] N. Zhang, B. Karp, S. Floyd, and L. Peterson, RR-TCP: A reorderingrobust TCP With DSACK. ICSI, Berkeley, CA, Tech. Rep. TR-02-006, Jul. 2002

[18] V. Paxson, "End-to-end Internet packet dynamics," presented at the ACM SIGCOMM, Cannes, France, 1997.

[19] S. Bhandarkar, N. Sadry, A. L. N. Reddy, and N. Vaidya, "TCP-DCR: a novel protocol for tolerating wireless channel errors," IEEE Trans. Mobile Comput., vol. 4, no. 5, pp. 517-529, Sep.-Oct. 2004.

[20] B. Sikdar, S. Kalyanaraman, and K. S. Vastola, "Analytic models for the latency and steady-state throughput of TCP Tahoe, Reno, and SACK," IEEE/ACM Trans. Netw., vol. 11, no. 6, pp. 959-971, Dec. 2003.

[21] M. Allman and V. Paxson, "Computing TCP's retransmission timer," RFC 2988, Nov. 2000.

[22] S. Bohacek, "A stochastic model of TCP and fair video transmission," in Proc. IEEE INFOCOM, 2003, pp. 1134-1144. 
[23] R. Braden, "Requirements for Internet Hostsdoctype - communication layers," RFC 1122, Oct. 1989.

[24] V. Jacobson, "Congestion avoidance and control," in ACM Comput. Commun. Rev.; Proc. SIGCOMM'88, Stanford, CA, Aug. 1988, vol. 18, no. 4, pp. 314-329.

[25] M. Allman and V. Paxson, "On estimating end-to-end network path properties," presented at the ACM SIGCOMM, Cambridge, MA, 1999.

[26] The VINT Project, a collaboration between researchers at UC Berkeley, LBL, USC/ISI, and Xerox PARC, The ns Manual (formerly $n s$ Notes and Documentation). [Online]. Available: http://www.isi.edu/nsnam/ns/ns-documentation.html, Oct. 2000

[27] M. Mathis, J. Mahdavi, S. Floyd, and A. Romanow, "TCP selective acknowledgment options," RFC 2018, 1996.

[28] S. Floyd, M. Handley, J. Padhye, and J. Widmer, "Equation-based congestion control for unicast applications," presented at the ACM SIGCOMM, Stockholm, Sweden, 2000.

[29] S. Floyd, "Connections with multiple congested gateways in packetswitched networks part 1: one-way traffic," ACM Comput. Commun. Rev., vol. 21, no. 5, pp. 30-47, Oct. 1991.

[30] D. Katabi, M. Handley, and C. Rohrs, "Internet congestion control for future high bandwidth-delay product environments," presented at the ACM SIGCOMM, Pittsburgh, PA, 2002.

[31] F. Hernández-Campos, J. S. Marron, G. Samorodnitsky, and F. D. Smith, "Variable heavy tail duration in Internet traffic," presented at the IEEE/ACM MASCOTS, Fort Worth, TX, 2002.

[32] S. Floyd, Scripts for Adaptive Red Simulations. [Online]. Available: http://www.icir.org/floyd/adaptivered/papersims/single1.tcl

[33] D. Chiu and R. Jain, "Analysis of the increase/decrease algorithms for congestion avoidance in computer networks," J. Comput. Netw. ISDN, vol. 17, pp. 1-14, 1989.

[34] S. Bohacek, J. Hespanha, K. Obraczka, and J. Lee, "Analysis of a TCP hybrid model," in Proc. 39th Annu. Allerton Conf. Communication, Control and Computing, Monticello, IL, 2001.

[35] M. Allman, H. Balakrishnan, and S. Floyd, "Enhancing TCP's loss recovery using limited transmit," RFC 3042, 2001

[36] J. Hespanha and S. Bohacek, "Preliminary results in routing games," presented at the American Control Conf., Arlington, VA, Jun. 2001.

[37] The TCP-PR Web Page. [Online]. Available: http://eecis.udel.edu/ bohacek/tcp-pr.htm

[38] B. Davie, A. Charny, J. C. R. Bennett, K. Benson, J. Y. Le Boudec, W. Courtney, S. Davari, V. Firoiu, and D. Stiliadis, "An expedited forwarding PHB (Per-Hop Behavior),” RFC 3246, 2001.

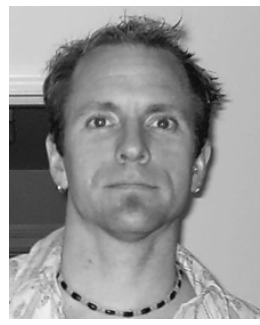

Stephan Bohacek (M'00) received the B.S. degree in electrical engineering from the University of California at Berkeley in 1989, and the Ph.D. degree in electrical engineering from the University of Southern California, Los Angeles, in 1999.

$\mathrm{He}$ is currently an Assistant Professor in the Department of Electrical and Computer Engineering at the University of Delaware, Newark. His research focuses on the design, analysis, and control of data networks. His current interests include congestion control and routing for wireless and wireline networks, modeling mobile wireless networks, and cross-layer design for wireless networks.

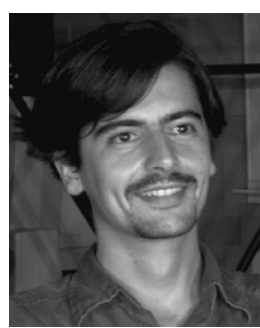

João P. Hespanha (S'95-A'98-SM'02) was born in Coimbra, Portugal, in 1968. He received the Licenciatura and the M.S. degree in electrical and computer engineering from the Instituto Superior Tecnico, Lisbon, Portugal, in 1991 and 1993, respectively, and the M.S. and Ph.D. degrees in electrical engineering and applied science from Yale University, New Haven, CT, in 1994 and 1998, respectively.

He is currently an Associate Professor in the Department of Electrical and Computer Engineering,
University of California at Santa Barbara. From 1999 to 2001, he was an Assistant Professor at the University of Southern California, Los Angeles. His research interests include switching control, hybrid systems, nonlinear control, both robust and adaptive, control of communication networks, the use of vision in feedback control, and probabilistic games. He is the author of over 100 technical papers.

Dr. Hespanha was the recipient of an NSF CAREER Award in 2001, and the PI and co-PI in several federally funded projects. Since 2003, he has been an Associate Editor of the IEEE TRANSACTIONS ON AUTOMATIC CONTROL. For his Ph.D. work, he received Yale University's Henry Prentiss Becton Graduate Prize for exceptional achievement in research in engineering and applied science.

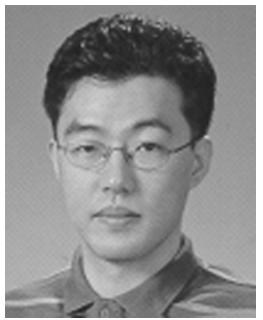

Junsoo Lee received the B.S. and M.S. degrees in computer science from Seoul National University, Seoul, Korea, and the Ph.D. degree in computer science from the University of Southern California, Los Angeles, in 2004.

He is currently an Assistant Professor of computer science at Sookmyung Women's University, Seoul, Korea. Previously, he was a Postdoctoral Scholar at UC Santa Barbara and UC Santa Cruz. His research interests include hybrid systems, wireless sensor networks, and wireless ad hoc networks.

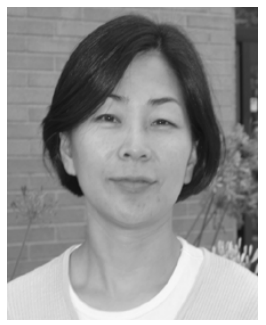

Chansook Lim (S'05) received the B.S. degree from Seoul National University, Seoul, Korea, and the M.S. degree from New York University, New York, NY. She is currently working toward the Ph.D. degree in computer science at the University of Southern California, Los Angeles.

Her research focuses on multi-path routing for robustness and design of transport protocols for multipath routing.

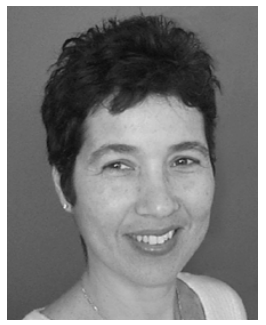

Katia Obraczka (S'90-M'92) received the B.S. and M.S. degrees in electrical and computer engineering from the Federal University of Rio de Janeiro, Brazil, and the M.S. and Ph.D. degrees in computer science from the University of Southern California (USC), Los Angeles.

She is currently an Associate Professor of computer engineering at the University of California, Santa Cruz (UCSC). Before joining UCSC, she held a reserach scientist position at USC's Information Sciences Institute and a research faculty appointment at USC's Computer Science Department. Her research interests include computer networks, more specifically, network protocol design and evaluation in wireline as well as wireless (in particular, multi-hop ad hoc) networks, distributed systems, and Internet information systems. She has been a PI and a co-PI in a number of projects sponsored by government agencies (NSF, DARPA, NASA) as well as industry (Cisco, Nokia, etc.). She has authored over 60 technical papers in journals and conferences.

Dr. Obraczka is currently on the Editorial Board of the Elsevier Ad Hoc Networks Journal. She has been Program Co-Chair for the IEEE Global Internet 2001 Symposium and helped organize several conferences, including the First IEEE Communications Society Conference on Sensor and Ad Hoc Communications and Networks (SECON 2004). She has also served on the program committees of several IEEE- and ACM-sponsored conferences. 